\title{
Simulations of Inter-Strip Capacitance and Resistance for the Design of the CMS Tracker Upgrade
}

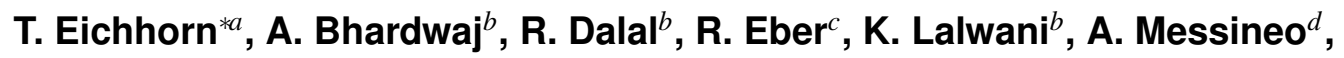 \\ T. Peltola ${ }^{e}$, M. Printz ${ }^{c}$ and K. Ranjan ${ }^{b}$ \\ ${ }^{a}$ Deutsches Elektronen-Synchrotron DESY (DE), ${ }^{b}$ University of Delhi (IN), ${ }^{c}$ Karlsruhe Institute \\ of Technology (DE), ' Università di Pisa \& INFN sez. di Pisa (IT), ${ }^{e}$ Helsinki Institute of Physics \\ $(F I)$
}

E-mail: thomas.eichhornedesy.de

\begin{abstract}
An upgrade of the LHC accelerator, the high luminosity phase of the LHC (HL-LHC), is foreseen for 2023. The tracking system of the CMS experiment at the HL-LHC will face a more intense radiation environment than the present system was designed for. This requires an upgrade of the full tracker, which will be equipped with higher granularity as well as radiation harder sensors, which can withstand higher radiation levels and occupancies.
\end{abstract}

In order to address the problems caused by the intense radiation environment, extensive measurements and simulation studies have been initiated for investigating these different design and material options for silicon micro-strip sensors.

The simulation studies are based on commercial packages (Silvaco and Synopsys TCAD) and aim to investigate sensor characteristics before and after irradiation for fluences up to $1.5 \cdot 10^{15} \mathrm{n}_{\mathrm{eq}} / \mathrm{cm}^{2}$. A defect model was developed to implement the radiation damage and tuned to fit experimental measurements.

This paper covers the simulation of the inter-strip capacitance and resistance both before and after irradiation. Both properties are crucial for the design of future sensors, being responsible for strip noise and isolation, in turn affecting resolution. A detailed understanding of these parameters is required for an optimal sensor design for the future CMS tracker.

Technology and Instrumentation in Particle Physics 2014,

2-6 June, 2014

Amsterdam, the Netherlands

\footnotetext{
* Speaker.
} 


\section{Introduction}

The currently installed silicon strip tracker in the CMS detector was designed for a running time of 10 years and a peak instantaneous luminosity of $10^{34} \mathrm{~cm}^{-2} \mathrm{~s}^{-1}$. The upcoming HL-LHC phase will not only increase the instantaneous luminosity by a factor of five, but also create an even harsher radiation environment. An upgraded tracker will therefore experience an increased particle flux and requires increased radiation hardness of sensors. It is planned to use information from the tracker in the first level CMS trigger and to additionally reduce the material budget. The design of new silicon sensors can be enhanced by the use of finite-element Technology Computer Aided Design (TCAD) simulations, which were performed with two commercially available software frameworks, Synopsys Sentaurus ${ }^{1}$ and Silvaco Atlas ${ }^{2}$.

The CMS Tracker Collaboration has started a campaign to identify not only properties and production processes of various silicon materials, but also to provide a baseline for a possible future sensor generation [1], complemented by device simulation studies. This ongoing campaign focuses on determining radiation damage effects and annealing behaviour, as well as evaluating sensor geometries and materials.

\section{Inter-Strip Capacitance and Resistance}

Two important strip sensor characteristics are the inter-strip capacitance $\mathrm{C}_{\mathrm{int}}$ and inter-strip resistance $\mathrm{R}_{\text {int }}$. They are defined as the capacitance or resistance of an individual strip towards its next neighbours. Since these properties contribute to strip noise and strip isolation, respectively, a detailed understanding of these parameters is necessary.

Within the above mentioned campaign, numerous measurements of $C_{i n t}$ and $R_{i n t}$ have been performed on dedicated multi-geometry silicon strip detector (MSSD) test sensors. The MSSD sensors have an active thickness from $120 \mu \mathrm{m}$ to $320 \mu \mathrm{m}$ and a strip pitch between $70 \mu \mathrm{m}$ and $240 \mu \mathrm{m}$. By varying the strip implantation width, three width to pitch ratios $\mathrm{w} / \mathrm{p}$ of $0.15,0.25$ and 0.33 have been realized, for n-type and p-type bulk material, the latter using $\mathrm{p}$-spray and also p-stop strip isolation technology. The strips have a length of $30490 \mu \mathrm{m}$. These measurements can be used as a benchmark to evaluate sensor simulation results. Also, as described in [2], TCAD simulations can be used to estimate unknown sensor geometries. $\mathrm{C}_{\text {int }}$ and $\mathrm{R}_{\mathrm{int}}$ are ideal properties to check against, as they are highly dependent on the sensor geometry.

The values for $\mathrm{C}_{\mathrm{int}}$ between two strips $i$ and $j$ were calculated with the following formula, in accordance with [3]:

$$
\mathrm{C}_{\text {int }}=\mathrm{C}_{\mathrm{AC}_{\mathrm{i}}-\mathrm{AC}_{\mathrm{j}}}+\mathrm{C}_{\mathrm{AC}_{\mathrm{i}}-\mathrm{DC}_{\mathrm{j}}}+\mathrm{C}_{\mathrm{DC}_{\mathrm{i}}-\mathrm{DC}_{\mathrm{j}}}+\mathrm{C}_{\mathrm{DC}_{\mathrm{i}}-\mathrm{AC}_{\mathrm{j}}}
$$

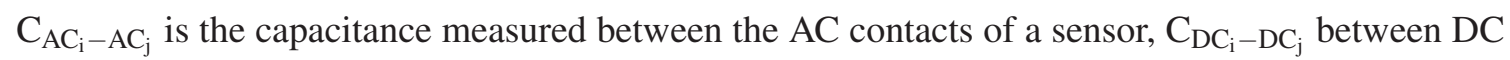
contacts. $\mathrm{C}_{\mathrm{AC}_{\mathrm{i}}-\mathrm{DC}_{\mathrm{j}}}$ refers to the capacitance between an $\mathrm{AC}$ and a $\mathrm{DC}$ contact. In the simulation,

\footnotetext{
${ }^{1}$ http://www.synospys.com

${ }^{2}$ http://www.silvaco.com
} 

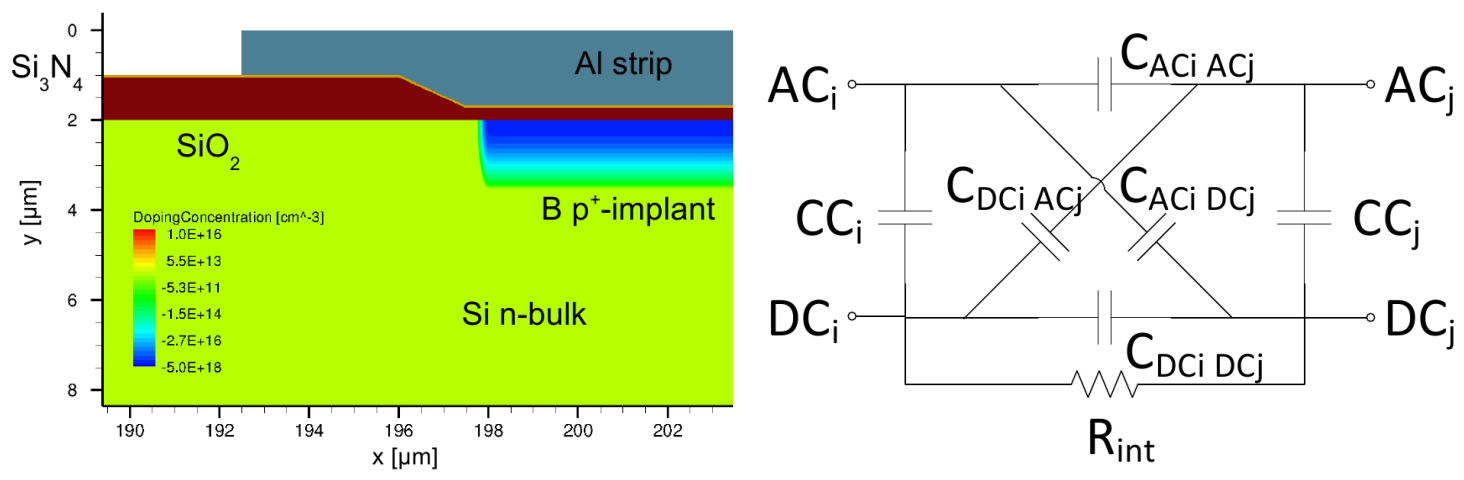

Figure 1: Left: Close-up of the simulated sensor structure. The silicon n-bulk is displayed in green, the boron $\mathrm{p}^{+}$-implant in blue. The aluminum read-out strip is grey, with the $\mathrm{SiO}_{2}$ in red. Note the additional layer of $\mathrm{Si}_{3} \mathrm{~N}_{4}$ in yellow. Right: measurement scheme for $\mathrm{C}_{\text {int }}$ and $\mathrm{R}_{\text {int }}$ between two strips with $\mathrm{AC}$ contacts $\mathrm{AC}_{\mathrm{i}}$ and $\mathrm{AC}_{\mathrm{j}}$ and $\mathrm{DC}$ contacts $\mathrm{DC}_{\mathrm{i}}$ and $\mathrm{DC}_{\mathrm{j}}$.

the AC contact was placed on top of the aluminum read-out strip and the DC contact on the strip implantation.

The inter-strip resistance $\mathrm{R}_{\text {int }}$ was simulated by biasing the sensor and, after reaching a steady state, applying one volt bias to a strip. The resistance was then calculated by measuring the resulting current on the neighbouring strips.
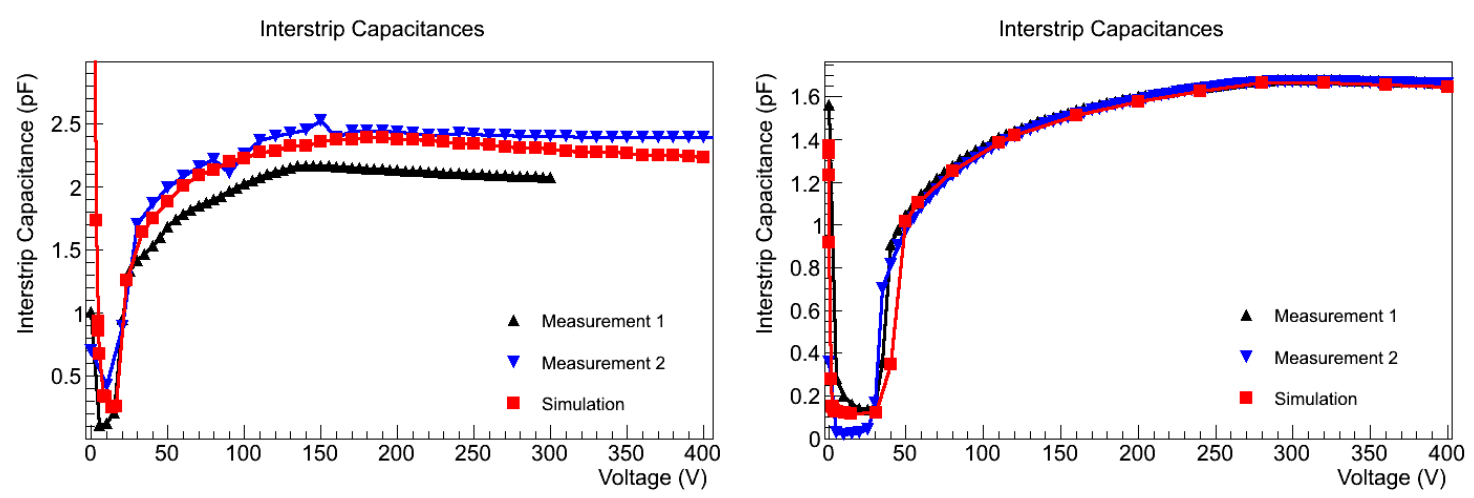

Figure 2: Left: $\mathrm{C}_{\text {int }}$ dependance on bias voltage for a $200 \mu \mathrm{m}$ thick p-type sensor with p-spray isolation and a pitch of $80 \mu \mathrm{m}$ before irradiation. The simulation in red is in agreement with measurements from different setups. Right: Simulated $C_{\text {int }}$ for a $320 \mu \mathrm{m}$ thick n-type sensor with a pitch of $120 \mu \mathrm{m}$ before irradiation, again in agreement with measurements.

In figure 2 two example simulations of $\mathrm{C}_{\mathrm{int}}$ are shown, for both $\mathrm{n}$ - and $\mathrm{p}$-bulk material, different thicknesses and pitch values. In both cases, the simulated results are in good agreement with the measurements. Simulated $\mathrm{C}_{\text {int }}$ vs. bias voltage curves also reproduce measurements for all other geometric MSSD variations. From this it can be concluded that the basic structure shown in figure 1 is a valid representation of the MSSD devices. Figure 3 shows simulated $R_{\text {int }}$ vs. bias voltage curves for a $200 \mu \mathrm{m}$ thick p-on-n sensor before irradiation, in agreement with the measurement values. 


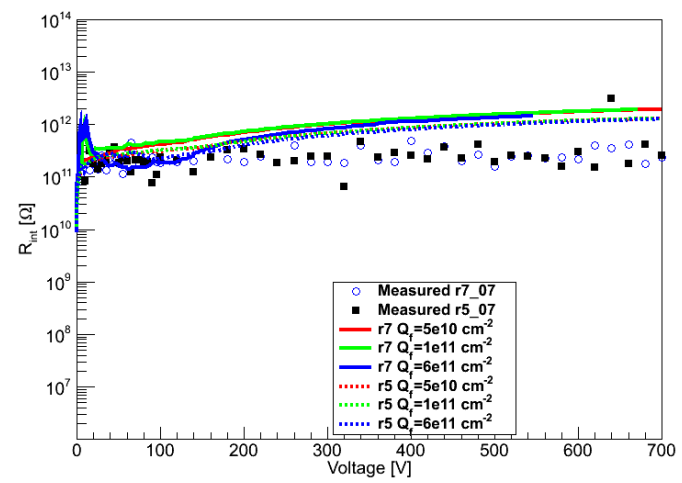

Figure 3: Inter-strip resistance $\mathrm{R}_{\mathrm{int}}$ dependance on bias voltage for different values of $\mathrm{Q}_{\mathrm{f}}$.

\section{Inter-Strip Capacitance and Inter-Strip Resistance for Irradiated Sensors}

Radiation damage is included in TCAD simulations by introducing traps, which are energy states in the silicon bandgap and can account for bulk damage. Surface damage is implemented by defining an interface charge $\mathrm{Q}_{\mathrm{f}}$ at the $\mathrm{Si}-\mathrm{SiO}_{2}$ interface. A bulk damage trap is defined by its energy $E$, concentration $c$ or introduction rate $\eta$ (with $\mathrm{c}=\eta \cdot \Phi, \Phi$ being the fluence) and its capture cross-sections $\sigma_{n}$ and $\sigma_{p}$ for electrons and holes, respectively. Depending on radiation type and simulation software there are several models in existence [4]. For fluences as high as expected for HL-LHC operating conditions, a combination of bulk and surface damage is necessary. The following simulations were performed with the model described in table 1 using Silvaco Atlas [5].

\begin{tabular}{|c||c|c|c|c|}
\hline Type & Energy in $\mathrm{eV}$ & $\sigma_{n}$ in $\mathrm{cm}^{2}$ & $\sigma_{p}$ in $\mathrm{cm}^{2}$ & $c$ in $\mathrm{cm}^{-3}$ \\
\hline \hline Acceptor & $E_{c}-0.525$ & $1 \cdot 10^{-14}$ & $1.4 \cdot 10^{-14}$ & $3 \cdot \Phi$ \\
\hline Acceptor & $E_{c}-0.45$ & $8 \cdot 10^{-15}$ & $2 \cdot 10^{-14}$ & $40 \cdot \Phi$ \\
\hline Acceptor & $E_{c}-0.40$ & $8 \cdot 10^{-15}$ & $2 \cdot 10^{-14}$ & $40 \cdot \Phi$ \\
\hline Donor & $E_{v}+0.50$ & $4 \cdot 10^{-15}$ & $4 \cdot 10^{-15}$ & $0.6 \cdot \Phi$ \\
\hline Donor & $E_{v}+0.45$ & $4 \cdot 10^{-15}$ & $4 \cdot 10^{-15}$ & $20 \cdot \Phi$ \\
\hline
\end{tabular}

Table 1: Used bulk damage model for proton irradiation with Silvaco Atlas.

After including radiation damage in the simulation, $\mathrm{C}_{\mathrm{int}}$ and $\mathrm{R}_{\text {int }}$ change, as is shown in figure 4 for various simulations and measured data. The inter-strip resistance drops to several G $\Omega$ and the voltage behaviour of $\mathrm{C}_{\text {int }}$ flattens out. As can be seen in both cases, it is essential to include surface damage in the form of an interface charge $\mathrm{Q}_{\mathrm{f}}$ alongside bulk damage to reproduce measurement values.

\section{Summary and Conclusion}

It has been shown that experimental measurements of inter-strip capacitance and resistance on MSSD sensors can be reproduced before and after irradiation by using an effective bulk damage 

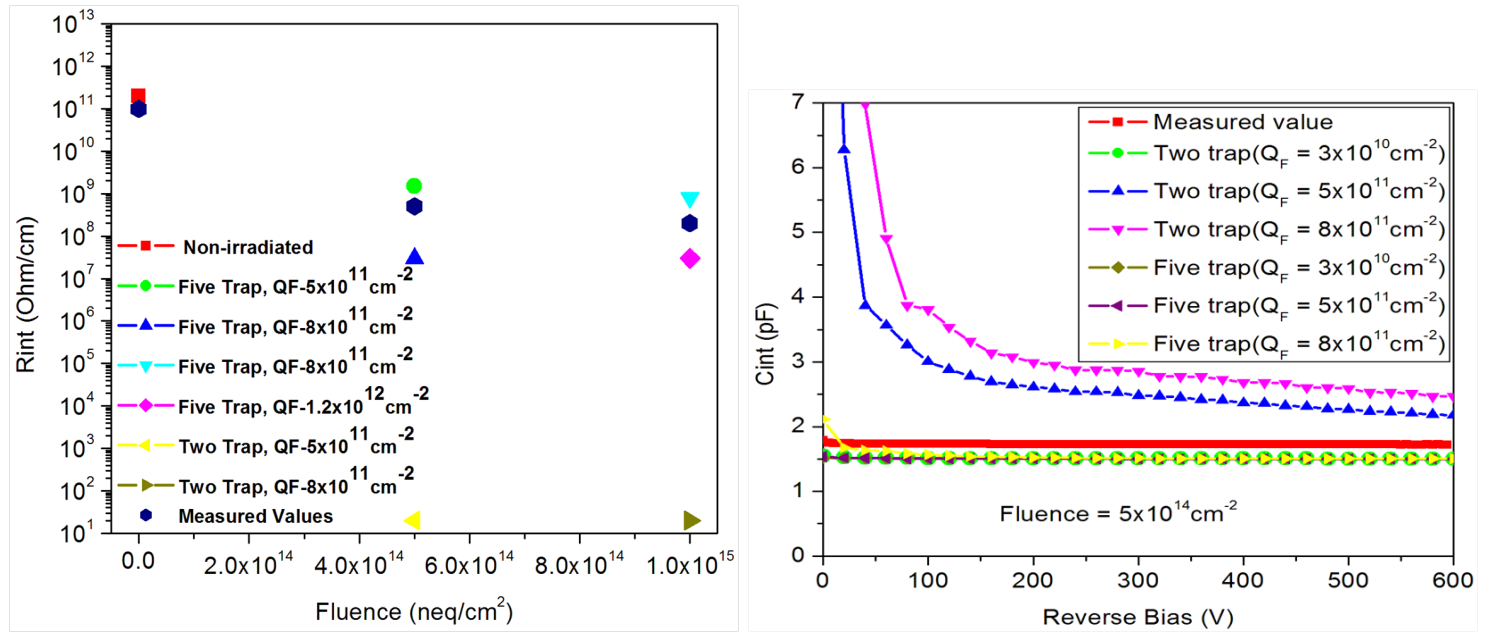

Figure 4: Left: $R_{\text {int }}$ for different fluences and values of $Q_{f}$. Right: Simulated $C_{i n t}$ for a $200 \mu m$ thick p-type sensor after irradiation with a fluence of $5 \cdot 10^{14} \mathrm{n}_{\mathrm{eq}} / \mathrm{cm}^{2}$.

model and including surface damage. Further work in this area [5] [6] shows that measurements of other sensor properties, such as leakage current, charge collection efficiency and depletion voltage can also be reproduced, both before and after including radiation damage. This could provide TCAD simulations with predictive power in contributing to the design of future sensors, not only for strip trackers but also for detectors in even higher radiation environments, such as pixel detectors.

\section{References}

[1] K.-H. Hoffmann, Campaign to identify the future CMS tracker baseline, Nucl. Instrum. Meth. A, 658(1):30 - 35, 2011, RESMDD 2010.

[2] T. Eichhorn, Silicon strip sensor simulations for the CMS phase-II tracker upgrade, IEEE, Nucl. Sci. Symp. (NSS/MIC) 2012, 1664-1669,

[3] S. Chatterji et al., Analysis of interstrip capacitance of Si microstrip detector using simulation approach, Solid-State Electronics, 47(1):1491 - 1499, 2003.

[4] R. Eber, Investigations of new Sensor Designs and Development of an effective Radiation Damage Model for the Simulation of highly irradiated Silicon Particle Detectors, PhD thesis (2013), Karlsruhe Institute of Technology, IEKP-KA/2013-27

[5] R. Dalal et al., Combined effect of bulk and surface damage on strip insulation properties of proton irradiated n+-p Silicon strip sensors, JINST 9 P04007, 2014

[6] T. Peltola et al., A method to model the accumulation of oxide charge with fluence in an irradiated MSSD, 24th RD50 Workshop, June, 11-13, 2014 Bucharest, Romania 